\title{
Mother's stress, mood and emotional involvement with the infant: 3 months before and 3 months after childbirth
}

\author{
Barbara Figueiredo $\cdot$ Raquel Costa
}

Received: 25 July 2008 /Accepted: 5 February 2009/Published online: 4 March 2009

(C) Springer-Verlag 2009

\begin{abstract}
Adverse effects of maternal anxiety and depression are well documented, namely on the foetus/child behaviour and development, but not as much attention has been given to the mother's emotional involvement with the offspring. To study mother's prenatal and postpartum stress, mood and emotional involvement with the infant, the StateTrait Anxiety Inventory, the Edinburgh Postnatal Depression Scale and the Mother-to-Infant Bonding Scale were filled in and cortisol levels were measured, 3 months before and 3 months after childbirth, in a sample of 91 Portuguese women. From pregnancy to the postpartum period, mother's cortisol levels, anxiety and emotional involvement toward the child decrease. No significant change was observed regarding mother's depression. Mother's depression predicted a worse emotional involvement before childbirth, while mother's anxiety predicted a worse emotional involvement with the infant after childbirth. Additionally, pregnant women with a worse emotional involvement with the offspring are at risk of poorer emotional involvement with the infant and higher anxiety and depression at 3 months postpartum. It should be given more attention to mother's poor emotional involvement with the offspring during pregnancy, as it interferes with her emotional involvement with the infant and her psychological adjustment 3 months after childbirth.
\end{abstract}

Keywords Anxiety C Cortisol - Depression . Mother's emotional involvement · Postpartum · Pregnancy

\footnotetext{
B. Figueiredo $(\bowtie) \cdot R$. Costa

Department of Psychology, Minho University,

Campus de Gualtar,

4710-057 Braga, Portugal

e-mail: bbfi@iep.uminho.pt

R. Costa

e-mail: rcosta@iep.uminho.pt
}

\section{Introduction}

Anxiety and depression in women, during both pregnancy and the postpartum period, have been matters of great concern. Research has shown several adverse impacts of the woman's psychological maladjustment during the transition to parenthood, namely, on the couple's relationship (e.g. Figueiredo et al. 2008; Lovisi et al. 2005; Pajulo et al. 2001) as well as on the foetal (e.g. DiPietro et al. 2002; Monk et al. 2000), neonatal (Field et al. 2004; Field et al. 2006), child (e.g. Van den Bergh and Marcoen 2004), and adolescent (Pawlby et al. 2008; Van den Bergh et al. 2005) development of the offspring. In the present study, we will focus particularly on the impact of mother's anxiety and depression, both before and after childbirth, on the emotional involvement with the infant, a dimension that is seldom studied.

Rates for anxiety and depression are high during pregnancy and the postpartum period, although a decrease in these rates, from the time before childbirth until after, has been noticed recently (e.g. Andersson et al. 2006; Buist et al. 2003; Chee et al. 2005; Figueiredo et al. 2006; Heron et al. 2004; Lee et al. 2007; Limlomwongse and Liabsuetrakul 2006; Perren et al. 2005). For example, anxiety and depression were prevalent in $29.2 \%$ of the pregnant women versus $16.5 \%$ of the postpartum women in a recent study by Andersson et al. (2006) with 1,555 Swedish women. Similarly, an increase during pregnancy and a decrease after childbirth in cortisol levels have also been reported in the literature (Field et al. 2004; Kammerer et al. 2006; Obel et al. 2005; Storey et al. 2000; Weerth and Buitelaar 2005). Elevated cortisol levels have also been found in women exhibiting psychological distress during pregnancy (Diego et al. 2006; Field et al. 2004; Field et al. 2006).

Empirical studies have reported high anxiety rates in more than $25 \%$ of the pregnant women (Britton 2005; 
Rondo et al. 2003; Ross et al. 2003; Ross and Mc Lean 2006; Sutter-Dalay et al. 2004). Anxiety levels seemed to be higher during pregnancy when compared with the postpartum period (Andersson et al. 2006; Buist et al. 2003; Heron et al. 2004; Lee et al. 2007). Additionally, studies account for depression in more than $15 \%$ of the pregnant women (Andersson et al. 2006; Chee et al. 2005; Dietz et al. 2007; Field et al. 2008; Figueiredo et al. 2007a; Edhborg et al. 2005; Marcus et al. 2003; Westdahl et al. 2007; Nicholson et al. 2006; Ross et al. 2004; Wu et al. 2002). Findings have shown these rates diminishing from the first to the third pregnancy trimester (Perren et al. 2005) and from pregnancy to the postpartum period (Andersson et al. 2006; Chee et al. 2005; Figueiredo et al. 2006; Heron et al. 2004; Limlomwongse and Liabsuetrakul 2006; Perren et al. 2005).

Developmental tasks throughout the transition to parenthood involve some important changes in the significant relationships of the mother and father, specifically with each other, with the extended family and with the offspring (Cowan and Cowan 2000). At first, mothers and fathers accept the pregnancy and the gestation of the child. Then, they begin imagining how the infant is/will be and how they will be/want to be as parents. So, they begin establishing a relationship with the infant (e.g. Ammaniti 1991; Stern 1995) as well as being emotionally involved with him/her (e.g. Rubin 1984). The mother and father's relationship with the offspring, which starts during pregnancy, has, from the beginning, an emotional dimensionalso called prenatal attachment (e.g. Cranley 1981; Shieh et al. 2001). The mother loves her unborn child through developmental tasks which include acceptance, incorporation, bonding and giving of herself (Bloom 1995; Rubin 1984). The mother and father become emotionally involved, as they progressively invest their emotions in the infant, as well as investing in the relationship with him/her. This will prepare them for the presence of the child in their real lives. First, they develop the mental space and the necessary emotional availability, so that afterwards they will receive the infant in their day-to-day life, have a relationship with and be attached to her/him.

Prenatal relationship and attachment facilitates the parents' initial reaction to the newborn (Cranley 1981; Reading et al. 1984; Shin et al. 2006), as well as the adequate interaction and care (Bloom 1995; Caccia et al. 1991), necessary to the child's survival (Bowlby 1958). However, other than the fact that the relationship between the mother and the infant begins well before childbirth, we do not know much about prenatal attachment (Fuller et al. 1993).

Maternal emotional involvement with the infant starts as early as the first weeks of gestation, and generally grows as the pregnancy progresses (Bloom 1995; Caccia et al. 1991; Grace 1989; Fleming et al. 1997a; Heidrich and Cranley
1989; Ji et al. 2005; Reading et al. 1984; Righetti et al. 2005; Rustico et al. 2005; Zachariah 1994). Particular events such as feeling the foetus's movements or seeing the foetus in an ultrasound exam seem to improve prenatal attachment (Ekelin et al. 2004; Ji et al. 2005; Kohn et al. 1980; Kowalcek et al. 2003; Lawson and Turriff-Jonasson 2006; Rustico et al. 2005). Ultrasound scans give the parents-to-be a clearer image of their unborn child, as their perception of the foetus evolves from that of an anonymous object into an individual (Kowalcek et al. 2003).

Nevertheless, some researchers have suggested that childbirth is the critical moment for maternal bonding, which is established from this instant (Klaus and Kennell 1976; Klaus et al. 1995). Authors noticed positive changes in the mother's emotional involvement with the offspring after delivery only (Fleming et al. 1997a), or a significant enhancement in the presence of the interaction with the child from delivery to 1 year postpartum (Moehler et al. 2006; Taylor et al. 2005). Additionally, some studies have shown that not all mothers are emotionally involved with the neonates immediately after childbirth, specifically after a painful or difficult delivery (Robson and Kumar 1980; Newton and Newton 1962). However, other studies observe that most mothers are emotionally involved with the newborn from the first days after delivery (Figueiredo et al. 2007b, 2009; Risk et al. 2001).

Several factors seem to interfere negatively with the mother's emotional involvement with the infant, particularly depression both before (Figueiredo et al. 2007a; Lindgren 2003) and after delivery (Edhborg et al. 2005; Feldman et al. 1999; Figueiredo et al. 2007c; Moehler et al. 2006; Nagata et al. 2003; Reck et al. 2006; Taylor et al. 2005). In spite of that, Honjo et al. (2003) did not find any association between mother's depression and attachment to the child in the first pregnancy trimester.

Adverse effects of anxiety on mother's emotional involvement with the child are not as obvious in the literature. During pregnancy, difficulties in the mother's emotional involvement with the offspring may be caused by stress (Cranley 1981; Hsu and Chen 2001). Several empirical evidences have demonstrated that parental anxiety diminishes after ultrasound exams, while prenatal attachment improves (Garcia et al. 2002; Segdmen et al. 2006). Simply seeing the infant or being reassured that he/ she is in good health (Garcia et al. 2002; Salisbury et al. 2003; Zlotogorski et al. 1995) may reduce parental anxiety and allow attachment to increase (Kleinveld et al. 2006, 2007; Segdmen et al. 2006). Both before and after childbirth, parental high anxiety regarding the infant's survival seems to interfere negatively with their availability to bond and invest the child emotionally (e.g. Feldman et al. 1999). Conversely, studies have reported higher cortisol levels in women related to elevated emotional involvement 
with the newborn (Fleming and Corter 1988; Fleming et al. 1997b; Giardino et al. 2008), but associations between these variables has not been as explored.

Both anxiety and depression are quite prevalent in women during pregnancy and the postpartum period (e.g. Andersson et al. 2006), with several documented negative effects on the offspring (e.g. Pawlby et al. 2008). However, the impact of anxiety and depression on the mother's emotional involvement with the infant is a dimension that has not been so thoroughly studied.

The purpose of this study was to examine the mother's cortisol levels, anxiety, depression and emotional involvement with the infant both before and after childbirth, specifically (1) differences in the mother's cortisol levels, anxiety, depression and emotional involvement with the infant between 3 months before childbirth and 3 months after and (2) predictors of the mother's emotional involvement with the infant considering mother's cortisol levels, anxiety and depression 3 months before and 3 months after childbirth.

According to previous literature, we will expect a decrease in mother's anxiety (e.g. Lee et al. 2007) as well as on mother's depression (e.g. Heron et al. 2004) and cortisol levels (e.g. Kammerer et al. 2006) while an increase in mother's emotional involvement with the offspring from before to after childbirth (e.g. Fleming et al. 1997a). We will also expect mother's depression (e.g. Edhborg et al. 2005) and anxiety (e.g. Hsu and Chen 2001) to interfere negatively while mother's cortisol levels (e.g. Giardino et al. 2008) to be positively associated with the mother's emotional involvement with the offspring, both before and after childbirth.

\section{Method}

Sample

The sample was composed of 91 primiparous women, aged between 16 and 40 years old [88.7\% between 19 and 39 years $($ mean $=27.2$ years $)]$. Almost all participants were Caucasian (98.9\%) and Catholic (90.7\%). Most mothers had completed compulsory education (grade 9) (79.5\%), were employed $(72.2 \%)$ and were married or living with the father-to-be $(88.7 \%)$. The majority of participants were living with their partner only $(80.2 \%)$, but some were either living with both the partner and other relatives (9.9) or living alone (9.9\%) (see Table 1). Most mothers had a caesarean $(47.2 \%)$ or an instrumental delivery $(15.3 \%)$, and only $37.5 \%$ had a vaginal delivery.

Half of the infants were female (51.5\%) and the other half male $(48.5 \%)$. They were born with a gestational age ranging from 34 to 40 weeks ( $6.2 \%$ had 37 or less), and their weight at birth ranged from $2,060 \mathrm{~g}$ to $3,970 \mathrm{~g}(9.0 \%$ below 2,500 $\mathrm{g})$.
Table 1 Socio-demographics

\begin{tabular}{lc}
\hline & Mothers $(n=91)(\%)$ \\
\hline Age (years) & \\
$16-18$ & 10.3 \\
$19-28$ & 49.5 \\
$29-39$ & 39.2 \\
$\geq 40$ & 1.0 \\
Education (grade) & \\
$<9$ & 20.5 \\
$9-12$ & 56.6 \\
$>12$ & 22.9 \\
Occupation & \\
Employed & 72.2 \\
Unemployed & 21.6 \\
Student & 4.2 \\
Housewife & 2.0 \\
Marital status & \\
Married & 59.8 \\
Living with partner & 28.9 \\
Single & 11.3 \\
Cohabitation status & \\
Only with the partner & 80.2 \\
With the partner and other relatives & 9.9 \\
Without the partner & \\
\hline
\end{tabular}

Procedures

This research was conducted according to the prevailing ethical principles. A specific approval request for this research was required and obtained from the Hospital Ethical Commission. The participants were randomly selected in an Obstetrics Outpatient Hospital Unit (Porto, Portugal), and the exclusion criteria were not reading or writing Portuguese and multiple gestations. The aims and the procedures of the study were explained to the contacted mothers, and $90 \%$ of them agreed to participate after signing an informed consent form. Between weeks 21 and 28 of gestation (3 months before childbirth), the participants answered socio-demographic questions, filled in the questionnaires and supplied a 24-h urine sample. All of these procedures were repeated 3 months after childbirth.

The urine samples were stored frozen at $-20^{\circ} \mathrm{C}$ and then analysed in the laboratory, using the cortisol ADVIA Centaur that consisted of a competitive immunoassay using direct chemoluminescent technology. The procedure followed was: (1) take a $20 \mathrm{~nL}$ sample; (2) add $50 \mathrm{~nL}$ of Lite reagent and $250 \mathrm{~nL}$ of Solid Fase and incubate at $37^{\circ} \mathrm{C}$ for $5 \mathrm{~min}$; (3) separate, aspirate and wash the recipient with reagent water; (4) add the same amount of acid and basic reagent to initiate the chemoluminescent reaction. Few 
samples $(1.0 \%)$ had to be excluded from the study due to insufficient $(\leq 600 \mathrm{ml}) 24$-h volume.

Measures

\section{State-trait anxiety inventory}

The State-Trait Anxiety Inventory (STAI, Spielberg et al. 1970) is composed of two 20-item self-report scales for measuring anxiety that differentiates between the temporary condition of "state anxiety" (STAI-S, anxiety in a specific situation) and the more general and long-standing quality of "trait anxiety" (STAI-T, anxiety as a general trait). This instrument has been administered in several studies, both during pregnancy (Austin et al. 2007; Kleinveld et al. 2006) and the postpartum period (Skari et al. 2002, 2006).

The Portuguese version used in this study showed good internal consistence (Trait and State Cronbach alpha $=0.87$ and 0.88 ). Authors advise using a score equal to or higher than 45 to screen high-anxiety states (Biaggio et al. 1976). Only STAI-S results will be reported here.

\section{Edinburgh postnatal depression scale}

The Edinburgh Postnatal Depression Scale (EPDS, Cox et al. 1987) is a self-report questionnaire composed of ten items scored in a four-point Likert scale (0-3) designed to assess pregnancy and postpartum depression. This scale addresses the intensity of depressive symptoms within the previous 7 days and has been administered in several studies, both with pregnant and postpartum women, including in Portugal (Areias et al. 1996a, 1996b; Augusto et al. 1996; Figueiredo et al. 2006; Figueiredo et al. 2007c; Gorman et al. 2004). Numerous studies have used this instrument, both during pregnancy (Eberhard-Gran et al. 2003; Heron et al. 2004; Pajulo et al. 2001; Rich-Edwards et al. 2006; Rich-Edwards et al. 2006; Ross et al. 2003) and/or the postpartum period (Eberhard-Gran et al. 2004; Limlomwongse and Liabsuetrakul 2006; Edhborg et al. 2005; Moehler et al. 2006; Taylor et al. 2005).

The EPDS Portuguese version showed good internal consistency (Cronbach alpha $=0.85$ ) and test-retest reliability (Spearman correlation $=0.75$ ) (Figueiredo et al. 2006, 2007c). A score equal to or higher than 10 was indicated to screen for a major depressive episode, with a sensibility of $65 \%$ and specificity of $96 \%$ (as EPDS cut-off 11 showed a sensibility of $45 \%$ and specificity of $96 \%$ and EPDS cut-off 12 a sensibility of $35 \%$ and a specificity of $96 \%$ ) (Areias et al. 1996a, b).

\section{Bonding scale}

The Bonding Scale - a validated and extended Portuguese version of the 'New Mother-to-Infant Bonding Scale'
(Taylor et al. 2005) - is a self-report scale composed of 11 items in a four-point Likert scale [from 0 (not at All) to 3 (very much)], aiming to assess the mother's emotional involvement with the infant (Figueiredo et al. 2005). Mothers are asked about the intensity of their emotions, in a time they felt closer to the infant. In the Portuguese version, three items were added (Mad, Sad and Fearful) to the original scale (composed of eight items: Loving, Resentful, Neutral or Felt Nothing, Joyful, Dislike, Protective, Disappointed and Aggressive).

In the Portuguese version, the scale is composed of three subscales. The 'Positive Bonding' includes three items (Loving, Protective and Joyful) and measures positive emotional involvement. A score equal to or higher than 3 in the Positive Bonding subscale means the mother presented elevated positive emotions toward the infant. The 'Negative Bonding' includes six items (Mad, Aggressive, Sad, Resentful, Dislike and Disappointed) and evaluates negative emotional involvement. Scores equal to or higher than 1 in the Negative Bonding subscale show the mother presented elevated negative emotions toward the child. The 'Bonding Not Clear' subscale includes two items (Fearful, Possessive and Neutral or Felt Nothing) and indicates the presence of emotions not clearly related to emotional involvement. A score equal to or higher than 2 in the Not Clear Bonding subscale means the mother strongly presented not clear emotions toward the infant (Figueiredo et al. 2005).

This instrument showed only reasonable scores of internal consistency (alpha of Cronbach $=0.61$ for the antenatal version and 0.75 for the postnatal version), as obtained with the original scale (alpha of Cronbach=0.71) (Taylor et al. 2005), but good test-retest reliability (Spearman correlation $=0.49 ; p<0.01$ ) (Figueiredo et al. 2005).

Statistical analysis

1. In order to study differences in cortisol levels of the mother between 3 months before childbirth and 3 months after, STAI-S, EPDS and Bonding mean, paired samples $t$ tests were conducted.

2. For the purpose of identifying predictors of cortisol levels, STAI-S, EPDS and Bonding scores of the mother 3 months before childbirth, several separate stepwise linear regression analyses were conducted. In the regression analyses performed to identify predictors of the mother's cortisol levels 3 months before childbirth, the independent variables entered were the STAI-S, EPDS and Bonding results from 3 months before childbirth. To identify predictors of mother's STAI-S results 3 months before childbirth, the independent variables entered were cortisol levels, EPDS 
and Bonding results at 3 months before childbirth. To identify predictors of mother's EPDS results 3 months before childbirth, the independent variables entered were cortisol levels, STAI-S and Bonding results at 3 months before childbirth. To identify predictors of mother's Bonding results 3 months before childbirth, the independent variables entered were cortisol levels, STAI-S and EPDS results 3 months before childbirth.

3. For the purpose of identifying 3 months after childbirth predictors of 3 months after childbirth mother's cortisol levels, STAI-S, EPDS and Bonding scores separate stepwise linear regression analyses were conducted. In the regression analyses performed to identify predictors of mother's cortisol levels 3 months after childbirth, entered independent variables were 3 months after childbirth STAI-S, EPDS and Bonding scores. To identify predictors of 3 months after childbirth mother's STAI-S results entered independent variables were 3 months after childbirth cortisol levels, EPDS and Bonding results. To identify predictors of 3 months after childbirth mother's EPDS results entered independent variables were 3 months after childbirth cortisol levels, STAI-S and Bonding results. To identify predictors of 3 months after childbirth mother's Bonding results entered independent variables were 3 months after childbirth cortisol levels, STAI-S and EPDS results.

4. For the purpose of identifying 3 months before and 3 months after childbirth predictors of 3 months after childbirth mother's cortisol levels, STAI-S, EPDS and Bonding results, separate hierarchical stepwise linear regression analyses were conducted. In the first model, entered independent variables were cortisol levels, STAI-S, EPDS and Bonding results 3 months before childbirth (as presented in 2). In the second model, independent variables were 3 months after childbirth cortisol levels, STAI-S, EPDS and Bonding results (as presented in 3) were added.

\section{Results}

Mother's stress, mood and emotional involvement with the infant: differences between 3 months before and 3 months after childbirth

Slightly over half of the pregnant women (51.7\%) had cortisol levels above normative values ( $\geq 90 \mu \mathrm{g} / 24 \mathrm{~h}$ ) 3 months before childbirth, while only $18.3 \%$ had these levels 3 months after delivery. One in four of the pregnant women had a STAI-S $\geq$ $45(25.0 \%)$ or an EPDS $\geq 10(26.7 \%)$ at the third pregnancy trimester, while $15.4 \%$ of the women had a STAI-S $\geq 45$ and $26.7 \%$ an EPDS $\geq 10$ at 3 months postpartum. Moreover, it was noticed that mother's cortisol levels and anxiety, but not mother's depression, decreased from 3 months before to 3 months after childbirth (see Table 2).

Furthermore, most pregnant and postpartum women showed to be emotionally involved with their offspring at a higher rate before than after delivery (as a Total Bonding $\geq 2.5$ was observed in $88.5 \%$ of the mothers before and in $73.1 \%$ of the mothers at 3 months postpartum). Also, a considerable number of mothers presented elevated positive emotions toward the infant (Positive Bonding $\geq 3$ ) during pregnancy $(50.6 \%)$, but no more than $1.5 \%$ at 3 months postpartum. More mothers showed elevated negative emotions toward the child (Negative Bonding $\geq 1$ ) 3 months before $(34.1 \%)$ than 3 months after delivery $(4.5 \%)$. Also, strong not clear emotions (Not clear
Table 2 Mother's cortisol, STAI-S, EPDS and Bonding 3 months before and 3 months after childbirth (paired sample $t$ test)

\begin{tabular}{|c|c|c|c|c|}
\hline & \multirow{2}{*}{$\begin{array}{l}3 \text { months before } \\
\text { Mean } \\
(\mathrm{SD})\end{array}$} & \multirow{2}{*}{$\begin{array}{l}3 \text { months after } \\
\text { Mean } \\
(\mathrm{SD})\end{array}$} & \multirow[t]{2}{*}{$t$} & \multirow[t]{2}{*}{$P$} \\
\hline & & & & \\
\hline Cortisol & $\begin{array}{l}100.31 \\
(38.58)\end{array}$ & $\begin{array}{c}61.26 \\
(50.52)\end{array}$ & 3.688 & 0.000 \\
\hline STAI-S & $\begin{array}{c}38.67 \\
(11.17)\end{array}$ & $\begin{array}{c}34.48 \\
(10.19)\end{array}$ & 2.019 & 0.049 \\
\hline EPDS & $\begin{array}{c}6.82 \\
(3.39)\end{array}$ & $\begin{array}{c}7.04 \\
(5.04)\end{array}$ & 0.855 & 0.396 \\
\hline Positive bonding & $\begin{array}{c}2.69 \\
(0.43)\end{array}$ & $\begin{array}{c}1.88 \\
(0.32)\end{array}$ & 17.629 & 0.000 \\
\hline Negative bonding & $\begin{array}{c}0.09 \\
(0.19)\end{array}$ & $\begin{array}{c}0.09 \\
(0.24)\end{array}$ & -0.223 & 0.825 \\
\hline Not clear bonding & $\begin{array}{c}0.74 \\
(0.52)\end{array}$ & $\begin{array}{c}0.70 \\
(0.50)\end{array}$ & 0.000 & 1.000 \\
\hline Total bonding & $\begin{array}{c}2.69 \\
(0.21)\end{array}$ & $\begin{array}{c}2.50 \\
(0.18)\end{array}$ & 8.443 & 0.000 \\
\hline
\end{tabular}


Table 3 Predicting variables for (A) 3 months before childbirth mother's cortisol, STAI-S, EPDS and Bonding (stepwise linear regression analysis) and (B) 3 months after childbirth mother's cortisol, STAI-S, EPDS and Bonding (stepwise linear regression analysis)

\begin{tabular}{|c|c|c|c|c|c|}
\hline & Model & $R^{2}$ & $B$ & $F$ & $P$ \\
\hline \multicolumn{6}{|c|}{ (A) 3 months before } \\
\hline Cortisol & $\mathrm{N} / \mathrm{S}$ & & & & \\
\hline STAI-S & EPDS & 0.576 & 0.759 & 74.803 & 0.000 \\
\hline EPDS & STAI-S & 0.576 & 0.759 & 74.803 & 0.000 \\
\hline Positive bonding & EPDS & 0.140 & -0.375 & 8.977 & 0.004 \\
\hline $\begin{array}{l}\text { Negative } \\
\text { bonding }\end{array}$ & EPDS & 0.124 & 0.352 & 7.757 & 0.007 \\
\hline $\begin{array}{l}\text { Not clear } \\
\text { bonding }\end{array}$ & EPDS & 0.114 & 0.337 & 7.047 & 0.010 \\
\hline $\begin{array}{l}\text { Total bonding } \\
\text { (B) } 3 \text { months after }\end{array}$ & EPDS & 0.276 & -0.526 & 20.994 & 0.000 \\
\hline Cortisol & $\mathrm{N} / \mathrm{S}$ & & & & \\
\hline \multirow[t]{2}{*}{ STAI-S } & EPDS & 0.662 & 0.722 & 70.509 & 0.000 \\
\hline & Bonding & 0.699 & -0.214 & 40.706 & 0.044 \\
\hline EPDS & STAI-S & 0.662 & 0.814 & 70.509 & 0.000 \\
\hline Positive bonding & $\mathrm{N} / \mathrm{S}$ & & & & \\
\hline $\begin{array}{l}\text { Negative } \\
\text { bonding }\end{array}$ & STAI-S & 0.289 & 0.538 & 14.661 & 0.000 \\
\hline $\begin{array}{l}\text { Not clear } \\
\text { bonding }\end{array}$ & STAI-S & 0.250 & 0.500 & 12.026 & 0.001 \\
\hline Total bonding & STAI-S & 0.301 & -0.549 & 15.530 & 0.000 \\
\hline
\end{tabular}

Bonding $\geq 2$ ) were present in only a few mothers, before $(2.3 \%)$ and after delivery (3.0\%). Additionally, the mother's emotional involvement with the infant decreased from pregnancy to 3 months after delivery as well. Although a decrease in both positive and total bonding results from pregnancy to 3 months postpartum was observed, no significant time differences were obtained for mother's negative and not clear emotions toward the offspring (see Table 2).

Predictors of mother's stress, mood and emotional involvement with the infant 3 months before and 3 months after childbirth

No significant predictors were identified for mother's cortisol levels, either 3 months before or 3 months after childbirth, when considering mother's anxiety, mood and emotional involvement with the infant. Similarly, cortisol was not a significant predictor for mother's anxiety, depression or emotional involvement with the offspring, during pregnancy or the postpartum period (see Table 3).

Mother's prenatal anxiety was associated with depression, since mother's prenatal depression predicted mother's anxiety 3 months before childbirth, which explains $57.6 \%$ of the variance. In the same way, mother's postnatal anxiety was also associated with depression, but mother's involvement with the infant was a significant predictor too: mother's postpartum anxiety was predicted by mother's depression and by worse emotional involvement with the infant 3 months after delivery, both explaining $69.9 \%$ of the variance (see Table 3 ). When considering variables from both 3 months before and 3 months after childbirth, mother's postnatal anxiety was predicted by mother's prenatal emotional involvement with the infant and postpartum depression: a worse emotional involvement with the offspring 3 months before and more depressive symptoms 3 months after childbirth predicted mother's postpartum anxiety, which explains $65.7 \%$ of the variance (see Table 4).

Mother's prenatal depression was associated with anxiety: higher levels of anxiety predicted more depressive symptoms 3 months before childbirth, explaining $57.6 \%$ of the variance. Similarly, mother's postpartum depression

Table 4 Three months before (before) and 3 months after (after) childbirth predicting variables for 3 months after childbirth mother's cortisol, STAI-S, EPDS and Bonding (hierarchical stepwise linear regression analysis)

\begin{tabular}{lllrrrr}
\hline & Model & $R^{2}$ & $R^{2}$ change & $B$ & $F$ & $P$ (Model 1) \\
\hline Cortisol & N/S (Model 2) \\
STAI-S & & & & & & \\
& Bonding (before) & 0.114 & 0.144 & -0.075 & 5.262 & 0.029 \\
EPDS & EPDS (after) & 0.657 & 0.535 & 0.792 & 32.644 & 0.500 \\
& Bonding (before) & 0.144 & 0.141 & -0.083 & 5.389 & 0.027 \\
Positive bonding & STAI-S (after) & 0.679 & 0.537 & 0.789 & 32.808 & 0.455 \\
Negative bonding & N/S & & & & 0.000 \\
& Bonding (before) & 0.291 & 0.291 & -0.243 & 13.526 & 0.001 \\
Not clear bonding & STAI-S (after) & 0.429 & 0.138 & 0.475 & 12.001 & 0.164 \\
Total bonding & STAI-S (after) & 0.159 & 0.159 & 0.399 & 6.254 & 0.009 \\
& Bonding (before) & 0.152 & 0.153 & 0.095 & 5.945 & 0.020 \\
\hline
\end{tabular}

Model 1: 3 months before (before) childbirth predicting variables. Model 2: 3 months after (after) childbirth predicting variables added 
was associated with anxiety as well: again, anxiety predicted depressive symptoms 3 months after childbirth, which explains $66.5 \%$ of the variance (see Table 3). When considering as predictors the variables from both 3 months before and 3 months after childbirth, the mother's postpartum depression was predicted by mother's prenatal emotional involvement with the offspring and mother's postpartum anxiety: worse emotional involvement with the offspring 3 months before childbirth and higher anxiety 3 months after childbirth predicted more postpartum depressive symptoms, which explains $67.9 \%$ of the variance (see Table 4).

During the antenatal period, mother's depression was associated with worse emotional involvement as well as with the presence of a less positive but also a more negative and a not clear emotional involvement with the offspring. As shown in Table 3, mother's prenatal depression predicted a poorer emotional involvement (explaining $27.6 \%$ of the variance), in addition to less positive emotions (explaining $14.0 \%$ of the variance), but more negative emotions (explaining $12.4 \%$ of the variance) and more not clear emotions (explaining $11.4 \%$ of the variance) toward the foetus 3 months before childbirth.

During the postnatal period, mother's anxiety was associated with a worse emotional involvement (explaining $30.1 \%$ of the variance) as well as with a more negative (anxiety predicted more negative emotions, explaining $28.9 \%$ of the variance) and a higher not clear emotional involvement with the infant 3 months after childbirth (anxiety predicted more not clear emotions, explaining $25.0 \%$ of the variance). Thus, none of the considered variables predicted mother's postnatal positive emotional involvement with the infant (see Table 3).

When considering the variables from both 3 months before and 3 months after childbirth as predictors (see Table 4), mother's prenatal emotional involvement was associated with both mother's postnatal emotional involvement-as a poorer emotional involvement with the infant 3 months before childbirth predicted a worse emotional involvement with the infant 3 months after childbirth, explaining 15.2\% of the variance - and mother's postnatal negative emotional involvement with the child-as mother's poorer emotional involvement with the infant 3 months before childbirth predicted more negative emotions toward the infant 3 months after childbirth, explaining $29.1 \%$ of the variance. Furthermore, mother's postnatal anxiety was associated with her emotional involvement with the infant 3 months after childbirth - as mother's anxiety predicted a worse emotional involvement with the infant 3 months after childbirth, explaining $29.0 \%$ of the variance, as well as with mother's postnatal negative emotions - since mother's anxiety predicted more negative emotions toward the infant 3 months after childbirth, explaining $42.9 \%$ of the variance, and not clear emotional involvement-as mother's state anxiety predicted more not clear emotions toward the infant 3 months after childbirth, explaining $15.9 \%$ of the variance. Therefore, none of the considered variables predicted mother's postnatal positive emotional involvement with the infant.

\section{Discussion and conclusion}

In this study, we observed that, despite the presence of anxiety and depression in many of the pregnant and postpartum women, most mothers were able to be emotionally involved with their offspring, both before and after delivery. Nevertheless, a decrease in mother's emotional involvement, cortisol levels and state anxiety were noticed from 3 months before childbirth to 3 months after, while no change was observed in depression.

High and subsequently lowered mother's anxiety from pregnancy to 3 months postpartum was also observed by others (e.g. Andersson et al. 2006; Buist et al. 2003; Heron et al. 2004; Lee et al. 2007), but the absence of a significant decline in mother's depression is contrary to what literature more recently informs (Andersson et al. 2006; Chee et al. 2005; Figueiredo et al. 2006; Heron et al. 2004; Limlomwongse and Liabsuetrakul 2006; Perren et al. 2005). Furthermore, the decrease in mother's cortisol levels from pregnancy to the postpartum period has been consistently reported in the literature (e.g. Field et al. 2004; Kammerer et al. 2006; Obel et al. 2005; Storey et al. 2000; Weerth and Buitelaar 2005), but an increase in the mother's emotional involvement with the infant was equally expected, according to previously published data (e.g. Fleming et al. 1997b; Taylor et al. 2005).

Presented results illustrate the mother's emotional involvement with the offspring since before childbirth as well as differences in mother's emotional involvement between the times before until after childbirth. Mother's emotional involvement and positive emotions toward the infant are higher 3 months before childbirth than 3 months after. Thus, results seem to point out that the "imaginary child" is more positively invested by the mother during pregnancy than the "real child" after delivery. Hypothetically, it is easier for the mother to express - namely positive - emotions before birth when the child is not already there. Conversely, the 3-monthold infant is somewhat less idealised, and the mother takes into account difficulties that may have occurred, as well as the child as he/she is, instead of as she imagined the child to be (inversely to what occurs during pregnancy). Therefore, at this stage emotions are not so extreme; they are mediated by the interaction, rendering the emotional involvement apparently poorer.

In the present study, and contrary to our initial expectations, state anxiety and depression did not predict cortisol levels of the mother, and cortisol levels did not predict the mother's 
emotional involvement with the child, neither 3 months before nor 3 months after childbirth. Therefore, these results do not support reported higher cortisol levels in both depressed/ anxious mothers (e.g. Field et al. 2006; Field et al. 2008) and mothers who are more emotionally involved with the child (e.g. Fleming and Corter 1988; Fleming et al. 1997b; Giardino et al. 2008). Cortisol awakening response and diurnal cortisol profiles has been studied in postpartum women, and high levels of cortisol awakening were related with adverse early life experiences (Gonzalez et al. 2009). However, studies did not report differences in daily cortisol levels, when pregnant or postpartum depressed or anxious women were compared to non-depressed or non-anxious women (Shea et al. 2007; Nierop et al. 2006). No differences obtained in mother's cortisol levels may be dependent of the method used (24-h urinary cortisol).

The mother's state anxiety is predicted by mother's depression as much as the reverse, both 3 months before and 3 months after childbirth, suggesting comorbid anxiety and depression in women during pregnancy and the postpartum period, as pointed in recent studies (e.g. Field et al. 2003; Heron et al. 2004; Wenzel et al. 2005). Moreover, mother's anxiety and depression, 3 months after childbirth, are also predicted by prenatal emotional involvement with the infant, which points out that pregnant women with a poor emotional involvement with the offspring are at risk for postpartum anxiety and depression. More than mother's prenatal anxiety or depression, poor emotional involvement with the offspring seems to predict anxiety and depression during the postpartum period, given empirical support to the supposed importance of prenatal attachment on the mother's psychological adjustment and quality of the interaction with and care of the infant after childbirth (e.g. Fuller et al. 1993; Kemp et al. 1990; Wiberg et al. 1989). In order to advance the understanding of women's world and interpersonal psychological difficulties during pregnancy and the postpartum period, more attention should be given to the context of the relationship with the child, established since pregnancy.

Previous studies have demonstrated a negative effect of mother's prenatal depression (e.g. Lindgren 2003) or anxiety (e.g. Cranley 1981; Hsu and Chen 2001; Garcia et al. 2002; Segdmen et al. 2006) on the mother's attachment with the foetus as well as a negative effect of the mother's postpartum depression (Edhborg et al. 2005; Feldman et al. 1999; Figueiredo et al. 2007b; Moehler et al. 2006; Nagata et al. 2003; Reck at al. 2006; Taylor et al. 2005) or anxiety (Feldman et al. 1999) on the mother's emotional involvement toward the child after delivery. Nevertheless, they did not generally consider these two relevant dimensions and periods together, that is anxiety and depression, and the prenatal and the postpartum period. The results of the present study give a more comprehensive picture of the dimensions implicated in the mother's difficulties to be emotionally involved with the infant, both before and after childbirth, providing specific cues for intervention. Besides, as has been more recently pointed out by other authors (e.g. Austin et al. 2007; Heron et al. 2004; Matthey et al. 2003), it is important to take into account both mother's anxiety and depression, when significant psychological difficulties during pregnancy and the postpartum period are targeted.

Acknowledgements We would like to thank the Human Development and Health Service of the Calouste Gulbenkian Foundation (Ref. 48914), the Bial Foundation (Ref. 58/02) and all the mothers that participated in this study.

\section{References}

Ammaniti M (1991) Maternal representations during pregnancy and early infant-mother interactions. Infant Ment Health J 12(3): 246-255

Andersson L, Sundström-Poromaa I, Wulff M (2006) Depression and anxiety during pregnancy and six months postpartum, a followup study. Acta Obstet Gynecol Scand 85(8):937-944

Areias ME, Kumar R, Barros H (1996a) Correlates of postnatal depression in mothers and fathers. Br J Psychiatry 169(1):36-41

Areias ME, Kumar R, Barros H (1996b) Comparative incidence of depression in women and men, during pregnancy and after childbirth. Validation of the Edinburgh Postnatal Depression Scale in Portuguese mothers. Br J Psychiatry 169(1):30-35

Augusto A, Kumar R, Calheiros JM (1996) Post-natal depression in an urban area of Portugal: comparison of childbearing women and matches controls. Psychol Med 26:135-141

Austin MP, Tully L, Parker G (2007) Examining the relationship between antenatal anxiety and postnatal depression. J Affect Disord 101(1-3):169-174

Biaggio AM, Natalicio L, Spielberger CD (1976) The development and validation of an experimental Portuguese form of the StateTrait Anxiety Inventory. In: Spielberger CD, Dias-Guerrero (eds) Cross-cultural research on anxiety. Hemisphere/Wiley, Washington, DC, pp 29-40

Bloom KC (1995) The development of attachment behaviors in pregnant adolescents. Nurs Res 44(5):284-289

Bowlby J (1958) The nature of the child's tie to his mother. Int J Psychoanal 39:350-373

Britton J (2005) Pre-discharge anxiety among mothers of well newborns: prevalence and correlates. Acta Paediatr 94(12):1771-1776

Buist A, Morse C, Durkin S (2003) Men's adjustment to fatherhood: implications for obstetric health care. J Obstet Gynecol Neonatal Nurs 32(2):172-180

Caccia N, Johnson JM, Robinson GE (1991) Impact of prenatal testing on maternal-fetal bonding, chorionic villus sampling versus amniocentesis. Am J Obstet Gynecol 16:1122-1125

Chee CY, Lee DT, Chong YL (2005) Confinement and other psychosocial factors in perinatal depression: a transcultural study in Singapore. J Affect Disord 89(1-3):157-166

Cowan CP, Cowan PA (2000) Preventive intervention: a couple perspective on the transmission of attachment patterns. In: Clulow C (ed) Adult attachment and couple psychotherapy: the secure base in practice and research. Brunner-Routledge, London, pp 62-82

Cox JL, Holden JM, Sagovsky R (1987) Detection of postnatal depression. Development of the 10-item Edinburgh postnatal depression scale. Br J Psychiatry 150:782-786 
Cranley MS (1981) Development of a tool for the measurement of maternal attachment during pregnancy. Nurs Res 30:281-284

Diego M, Jones NA, Field T (2006) Prenatal cortisol mediates the effects of maternal psychological distress on fetal weight. Psychosom Med 68:747-753

Dietz PM, Williams SB, Callaghan WM (2007) Clinically identified maternal depression before, during, and after pregnancies ending in live births. Am J Psychiatry 164:1515-1520

DiPietro JA, Hilton SC, Hawkins M (2002) Maternal stress and affect influence fetal neurobehavioral development. Dev Psychol 38 (5):659-668

Eberhard-Gran M, Tambs K, Opjordsmoen S (2003) A comparison of anxiety and depressive symptomatology in postpartum and nonpostpartum mothers. Soc Psychiatry Psychiatr Epidemiol 38 (10):551-556

Eberhard-Gran M, Tambs K, Opjordsmoen S (2004) Depression during pregnancy and after delivery: a repeated measurement study. J Psychosom Obstet Gynaecol 25(1):15-21

Edhborg M, Matthiesen AS, Lundh W (2005) Some early indicators for depressive symptoms and bonding 2 months postpartum-a study of new mothers and fathers. Arch Womens Ment Health 8 (4):221-231

Ekelin M, Crang-Svalenius E, Dykes AK (2004) A qualitative study of mothers' and fathers' experiences of routine ultrasound examination in Sweden. Midwifery 20(4):335-344

Feldman R, Weller A, Leckman JF (1999) The nature of the mother's tie to her infant, maternal bonding under conditions of proximity, separation and potential loss. J Child Psychol Psychiatry 406:929-939

Field T, Diego M, Hernandez-Reif M (2003) Pregnancy anxiety and comorbid depression and anger effects on the fetus and neonate. Depress Anxiety 17:140-151

Field T, Diego M, Dieter J (2004) Prenatal depression effects on the fetus and neonate. Infant Behav Dev 27:216-229

Field T, Hernandez-Reif M, Diego M (2006) Prenatal cortisol, prematurity and low birthweight. Infant Behav Dev 29:268-275

Field T, Diego M, Hernandez-Reif M (2008) Chronic prenatal depression and neonatal outcome. Int J Neurosci 118(1):95-103

Figueiredo B, Marques A, Costa R (2005) Bonding: escala para avaliar o envolvimento emocional dos pais com o bebé. Psychologica 40:133-154

Figueiredo B, Bifulco A, Pacheco A (2006) Teenage pregnancy, attachment style and depression: a comparison of teenage and adult pregnant women in a Portuguese series. Attach Hum Dev 8 (2):123-138

Figueiredo B, Costa R, Pacheco A (2007a) Anxiété, dépression et investissement émotionnel de l'enfant pendant la grossesse. Devenir 19(3):243-260

Figueiredo B, Costa R, Pacheco A (2007b) Mother-to-infant and father-to-infant initial emotional involvement. Early Child Dev Care 177(5):521-532

Figueiredo B, Pacheco A, Costa R (2007c) Depression during pregnancy and the postpartum period in adolescent and adult Portuguese mothers. Arch Womens Ment Health 10(3):103-109

Figueiredo B, Field T, Diego M (2008) Partner relationships during the transition to parenthood. J Reprod Infant Psychol 26(2):99107

Figueiredo B, Costa R, Pacheco A et al (2009) Mother-to-infant emotional involvement at birth. Matern Child Health J 13 (3): in press

Fleming AS, Corter CM (1988) Factors influencing maternal responsiveness in humans: usefulness of an animal model. Psychoendocrinology 13:189-212

Fleming AS, Ruble D, Krieger H (1997a) Hormonal and experiential correlates of maternal responsiveness during pregnancy and the puerperium in human mothers. Horm Behav 31(2):145-58
Fleming AS, Steiner M, Corter CM (1997b) Cortisol, hedonics, and maternal responsiveness in human mothers. Horm Behav 32(2):85-98

Gonzalez A, Jenkins JM, Steiner M, Fleming AS (2009) The relation between early life adversity, cortisol awakening response and diurnal salivary cortisol levels in postpartum women. Psychoneuroendocrinology 34(1):76-86

Fuller SG, Moore LR, Lester JW (1993) Influence of family functioning on maternal-fetal attachment. J Perinatol 13(6): 453-460

Garcia J, Bricker L, Menderson J (2002) Women's views of pregnancy ultrasound: a systematic review. Birth 29(4):225-250

Giardino J, Gonzalez A, Steiner M (2008) Effects of motherhood on physiological and subjective responses to infant cries in teenage mothers: a comparison with non-mothers and adult mothers. Horm Behav 53:149-158

Gorman L, O'Hara M, Figueiredo B (2004) Adaptation of the Structured Clinical Interview for DSM-IV disorder for assessing depression in women during pregnancy and postpartum across countries and cultures. Br J Psychiatry 184(suppl.46):18-25

Grace JT (1989) Development of maternal-fetal attachment during pregnancy. Nurs Res 38(4):228-232

Heidrich SM, Cranley MS (1989) Effect of fetal movement, ultrasound scans, and amniocentesis on maternal-fetal attachment. Nurs Res 38(2):81-84

Heron J, O'Connor T, Evans J (2004) The course of anxiety and depression through pregnancy and the postpartum in a community sample. J Affect Disord 80(1):65-73

Honjo S, Arai S, Kaneko H (2003) Antenatal depression and maternal-fetal attachment. Psychopathology 36(6):304-311

Hsu TL, Chen CH (2001) Stress and maternal-fetal attachment of pregnant women during their third trimester. Kaohsiung $\mathrm{J}$ Med Sci 17(1):36-45

Ji E, Pretorious DM, Newton R (2005) Effects of ultrasound on maternal-fetal bonding: a comparison of two- and three dimensional imaging. Ultrasound Obstet Gynecol 25:473-477

Kammerer M, Taylor A, Glover V (2006) The HPA axis and perinatal depression: a hypothesis. Arch Womens Ment Health 9(4):187196

Kemp V, Sibley D, Pond E (1990) A comparison of adolescent and adult mothers on factors affecting maternal role attainment. Matern Child Nurs J 19(1):579-584

Klaus M, Kennel J (1976) Maternal-infant bonding. C.V. Mosby, Saint Louis

Klaus MH, Kennell JH, Klaus PH (1995) Bonding: building the foundations of secure attachment and independence. AddisonWesley, New York

Kleinveld JH, Timmermans DR, de Smit DJ, Adér HJ, van der Wal G, ten Kate LP (2006) Does prenatal screening influence anxiety levels of pregnant women? A longitudinal randomised controlled trial. Prenat Diagn 26(4):354-361

Kleinveld JH, Timmermans DR, van den Berg M, van Eijk JT, Ten Kate LP (2007) Does offering and performing prenatal screening influence women's attachment to their unborn child? A longitudinal randomized controlled trial. Prenat Diagn 27(8):757-764

Kohn CL, Nelson A, Weiner S (1980) Gravidas' responses to realtime ultrasound fetal image. J Obstet Gynecol Neonatal Nurs 9(2):77-80

Kowalcek I, Huber G, Bieniakiewitz I (2003) The influence of gestational age on depressive reactions, stress and anxiety of pregnant women and their partners in relation to prenatal diagnosis. J Psychosom Obstet Gynaecol 24(4):239-245

Lawson KL, Turriff-Jonasson SI (2006) Maternal serum screening and psychosocial attachment to pregnancy. J Psychosom Res 60 (4):371-378

Lee AM, Lam SK, Sze Mun Lau SM et al (2007) Prevalence, course, and risk factors for antenatal anxiety and depression. Obstet Gynecol 110:1102-1112 
Limlomwongse N, Liabsuetrakul T (2006) Cohort study of depressive moods in Thai women during late pregnancy and 6-8 weeks of postpartum using the Edinburgh Postnatal Depression Scale (EPDS). Arch Womens Ment Health 9(3):131-138

Lindgren K (2003) A comparison of pregnancy health practices of women in inner-city and small urban communities. J Obstet Gynecol Neonatal Nurs 32(3):313-321

Lovisi GM, Lopez JR, Coutinho ES (2005) Poverty, violence and depression during pregnancy, a survey of mothers attending a public hospital in Brazil. Psychol Med 35(10):1485-1492

Marcus SM, Flynn HA, Blow FC (2003) Depressive symptoms among pregnant women screened in obstetrics settings. J Womens Health 12(4):373-380

Matthey S, Barnett B, Howie P (2003) Diagnosing postpartum depression in mothers and fathers: whatever happened to anxiety. J Affect Disord 74(2):139-147

Möhler E, Brunner R, Wiebel A (2006) Maternal depressive symptoms in the postnatal period are associated with long-term impairment of mother-child bonding. Arch Womens Ment Health 9(5):273-278

Monk C, Fifer WP, Sloan RP (2000) Maternal stress responses and anxiety during pregnancy: effects on fetal heart rate. Dev Psychobiol 36:67-77

Nagata M, Nagai Y, Sobajima H (2003) Depression in the mother and maternal attachment - results from a follow-up study at 1 year postpartum. Psychopathology 36(3):142-151

Newton N, Newton M (1962) Mothers' reactions to their newborn babies. JAMA 21(181):206-210

Nicholson W, Setse R, Hill-Briggs F (2006) Depressive symptoms and health-related quality of life in early pregnancy. Obstet Gynecol 107:798-806

Nierop A, Bratsikas A, Zimmermann R (2006) Are stress-induced cortisol changes during pregnancy associated with postpartum depressive symptoms. Psychosom Med 68(6):931-937

Obel C, Hedegaard M, Henriksen TB (2005) Stress and salivary cortisol during pregnancy. Psychoneuroendocrinology 30 (7):647-656

Pajulo M, Savonlahti E, Sourander A (2001) Antenatal depression, substance dependency and social support. J Affect Disord 65:9

Pawlby S, Sharp D, Hay D (2008) Postnatal depression and child outcome at 11 years: the importance of accurate diagnosis. J Affect Disord 107(1-3):241-245

Perren S, Wyl A, Bürgin D (2005) Intergenerational transmission of marital quality across the transition to parenthood. Fam Process 44(4):441-459

Reading AE, Cox DN, Sledmere CM (1984) Psychological changes over the course of pregnancy: a study of attitudes toward the fetus/neonate. Health Psychol 3(3):211-221

Reck C, Klier CM, Pabst K (2006) Mother-infant bonding disorders in patients with postnatal depression: the Postpartum Bonding Questionnaire in clinical practice. Arch Womens Ment Health 9 (5):289-291

Rich-Edwards JW, Kleinman K, Abrams A (2006) Sociodemographic predictors of antenatal and postpartum depressive symptoms among women in a medical group practice. J Epidemiol Community Health 60:221-227

Righetti PL, Dell'Avanzo M, Grigio M (2005) Maternal/paternal antenatal attachment and fourth-dimensional ultrasound technique, a preliminary report. Br J Psychiatry 96:129-137

Rizk DE, Nasse M, Thomas L (2001) Women's perceptions and experiences of childbirth in United Arab Emirates. J Perinat Med 29(4):298-307

Robson KS, Kumar R (1980) Delayed onset of maternal affection after childbirth. Br J Psychiatry 136:347-353

Rondo PH, Ferreira RF, Nogueira F (2003) Maternal psychological stress and distress as predictors of low birth weight, prematurity and intrauterine growth retardation. Eur J Clin Nutr 57(2): 266-272

Ross LE, McLean LM (2006) Anxiety disorders during pregnancy and the postpartum period: a systematic review. J Clin Psychiatry 67 (8): 1285-1298

Ross LE, Gilbert Evans SE (2003) Measurement issues in postpartum depression part 1 , anxiety as a feature of postpartum depression. Arch Womens Ment Health 6(1):51-57

Ross LE, Sellers EM, Gilbert Evans SE (2004) Mood changes during pregnancy and the postpartum period: development of a biopsychosocial model. Acta Psychiatr Scand 109(6):457-466

Rubin R (1984) Maternal identity and the maternal experience. Springer, New York

Rustico MA, Mastromatteo C, Grigio M (2005) Two-dimensional vs. two- plus four-dimensional ultrasound in pregnancy and the effect on maternal emotional status, a randomized study. Ultrasound Obstet Gynecol 25:468-472

Salisbury A, Yanni P, Bocanegra M (2003) Fetal behavioral reactivity and depressed maternal mood. Arch Womens Ment Health 6 (suppl.1):s1-s28

Segdmen B, McMahon C, Cairns D (2006) The impact of twodimensional vs three-dimensional ultrasound exposure on maternalfetal attachment and maternal health behavior in pregnancy. Ultrasound Obstet Gynecol 27:245-251

Shea AK, Streiner DL, Fleming A (2007) The effect of depression, anxiety and early life trauma on the cortisol awakening response during pregnancy: preliminary results. Psychoneuroendocrinology 32(8-10): 1013-1020

Shieh C, Kravitz M, Wang H (2001) What do we know about maternal-fetal attachment. Kaohsiung J Med Sci 17:448-454

Shin H, Park Y, Kim MJ (2006) Predictors of maternal sensitivity during the early postpartum period. J Adv Nurs 55(4):425-434

Skari H, Skreden M, Malt UF (2002) Comparative levels of psychological distress, stress symptoms, depression and anxiety after childbirth - a population-based study. I Br J Obstet Gynaecol 109(10):1154-1163

Spielberg C, Gorsuch RL, Lushene RE (1970) The State-Trait Anxiety Inventory. Consulting Psychologists Press, Palo Alto

Stern D (1995) The motherhood constellation. Harper Collins, New York

Storey AE, Walsh CJ, Quinton RL (2000) Hormonal correlates of paternal responsiveness in new and expectant fathers. Evol Hum Behav 21(2):79-95

Sutter-Dallay AL, Giaconne-Marcesche V, Glatigny-Dallay E (2004) Women with anxiety disorders during pregnancy are at increased risk of intense postnatal depressive symptoms, a prospective survey of the MATQUID cohort. Eur Psychiatry 19 (8):459-463

Taylor A, Atkins R, Kumar R (2005) Mother-to-infant bonding scale: links with early maternal mood. Arch Womens Ment Health $81: 45-51$

Van den Bergh B, Marcoen A (2004) High antenatal maternal anxiety is related to ADHD symptoms, externalizing problems, and anxiety in 8- and 9-year-olds. Child Dev 75(4): $1085-1097$

Van den Bergh B, Mennes M, Oosterlaan J (2005) High antenatal maternal anxiety is related to impulsivity during performance on cognitive tasks in 14- and 15-year-olds. Neurosci Biobehav Rev 29(2):259-269

Weerth C, Buitelaar JK (2005) Cortisol awakening response in pregnant women. Psychoneuroendocrinology 30:902-907

Wenzel A, Haugen EN, Jackson LC (2005) Anxiety disorders at eight weeks postpartum. J Anxiety Disord 19:295-311

Westdahl C, Milan S, Magriples U (2007) Social support and social conflict as predictors of prenatal depression. Obstetrics Gynecology 110(1):134-140 
Wiberg B, Humble K, de Château P (1989) Long-term effect on mother-infant behaviour of extra contact during the first hour post partum. V Follow-up at three years. Scand J Soc Med 17:181-191

Wu JM, Viguera A, Riley L (2002) Mood disturbance during pregnancy is not associated with mode of delivery. Am J Obstet Gynecol 187:864
Zachariah R (1994) Maternal-fetal attachment: influence of motherdaughter and husband-wife relationships. Res Nurs Health 17:37-44

Zlotogorski Z, Tadmor O, Duniec E (1995) Anxiety levels of pregnant women during ultrasound examination, coping styles, amount of feedback and learned resourcefulness. Ultrasound Obstet Gynecol $6: 425-429$ 